\title{
La propuesta de identificación de la ciudad de Confloenta/Confluenta en Duratón (Sepúlveda, Segovia, Hispania Citerior)
}

\author{
Santiago Martínez Caballero \\ Museo de Segovia \\ marcabsa@jcyl.es \\ Julio Mangas ManjarRÉS \\ Universidad Complutense de Madrid \\ juliomangas@yahoo.es
}

Recibido: 17 de octubre de 2013

Aceptado: 7 de enero de 2014

\section{RESUMEN}

Se evalúa la propuesta de identificación de la Confloenta/Confluenta arévaca de Ptolomeo con la ciudad romana documentada por la arqueología en el yacimiento de Los Mercados de Duratón (Sepúlveda, Segovia), ciudad fundada a inicios del s. I a.C., en el marco de la política de urbanización republicana romana de la Celtiberia, y sede de un municipium de derecho latino desde época flavia.

Palabras clave: Confloenta. Confluenta. Duratón. Los Mercados. Sepúlveda. Celtiberia. Fundación. Municipium.

\section{The identification proposal for the city of Confloenta/Confluenta in Duratón (Sepúlveda, Segovia, Hispania Citerior)}

\begin{abstract}
This paper analyzes Ptolemy's identification proposal for the Arevaci Confloenta/Confluenta with the Roman city documented by archaeology in the Los Mercados site in Duratón (Sepúlveda, Segovia), founded at the start of the $1^{\text {st }}$ century B.C., as part of the Roman republican urbanisation policy of the Celtiberia, and the seat of a flavian municipium.
\end{abstract}

Key words: Confloenta. Confluenta. Duratón. Los Mercados. Sepúlveda. Celtiberia. Foundation. Municipium.

Sumario: 1. El origen de la identificación de Confluenta con Duratón. 2. Identificación del municipium de Duratón desde las fuentes epigráficas. 3. Identificación de Confluenta con el municipium de Duratón: consolidación del argumento arqueológico y revisión del argumento literario. 4. Conclusiones. 
Las investigaciones que hemos llevado a cabo desde los años 2000 en el yacimiento de Los Mercados (Duratón, Sepúlveda, Segovia) confirman la presencia en el lugar de una ciudad romana donde se observan parámetros urbanísticos y arquitectónicos propios de los enclaves urbanos romanos del valle del Duero de importancia regional y con estatuto jurídico privilegiado. De forma progresiva hemos ido generando una documentación que nos ha permitido proponer, con argumentos sólidos, la identificación de Duratón con la Confloenta de Ptolomeo 2.6.55, construcción de una hipótesis a la que atenderemos en lo sucesivo. ${ }^{1}$

\section{El origen de la identificación de Confluenta con Duratón}

No es hasta fines del s. XVIII cuando se formula la primera propuesta de identificación de Duratón con Confloenta, por J. Córnide, ${ }^{2}$ tras la intervención que en 1795 el arquitecto Juan de Villanueva acometió en Los Mercados (que deparó la extracción de varios mosaicos que luego habrían de decorar la Casa del Ermitaño del Palacio de Aranjuez). ${ }^{3}$ Otros autores recogerán esta propuesta entre los siglos XIX y principios del XX. ${ }^{4}$ Atendiendo a las coordenadas de Ptolomeo, que la sitúan al norte de Clunia,

1 Martínez Caballero 2000; Martínez Caballero - Mangas Manjarrés - Prieto Vázquez 2001; Martínez Caballero - Mangas Manjarrés 2002; Martínez Caballero - Prieto Vázquez 2002; Orejas Saco del Valle - Martínez Caballero 2002; Martínez Caballero - Prieto Vázquez - Orejas Saco del Valle 2004; Martínez Caballero 2008; ID. 2010; Mangas Manjarrés - Martínez Caballero 2010; Martínez Caballero - Santiago Pardo 2010; Martínez Caballero 2011; ID. 2011a; ID. 2013; ID. 2014; Santos Yanguas - Martínez Caballero 2014; Martínez Caballero 2014a; ID. e.p.; Martínez Caballero - López Ambite - Gallego Revilla e.p.

2 J. Córnide, apud Gómez de Somorrostro 1820, 220. También había propuesto su ubicación en Las Berlangas (Burgos), junto a Roa de Duero, en la confluencia del Abisejo (el Riaza) y el Duero (en el Informe de las Antigüedades de Cabeza del Griego; CóRnIDE 1799). Rui Wamba la colocaba entonces (texto inédito) en la confluencia de los ríos Arlanzón y Pisuerga. Loperráez 1788, 262, solo había señalado que confinaba con los vacceos. Con precedencia, RodríguEz VALCÁRCEL 1647, la identificaba con Fontiveros (Ávila). Sobre la localización de Confloenta, su identificación con Duratón, y su relación con la propuesta (errónea) de situar en la vecina Sepúlveda una ciudad romana: Martínez CABAllero 2014. Sepúlveda había sido identificada por Ginés de SEPÚlveda 1571, Epist. 37, Lib. 3, con Segobriga (propuesta recogida luego por varios autores: Grutero 1603, 90, nº 7; VAILlant 1695, 80-81), hipótesis luego rechazada desde trabajos como los de TraGGIA 1757, 297, y MaYANS $1779,145, n^{\circ} 93$. Los primeros datos modernos sobre el yacimiento de Los Mercados de Duratón provienen de Morales 1575, 60 y 193.

3 Las excavaciones, dirigidas en obra por los oficiales Antonio Febrer y "D. Vicente", son conocidas por las informaciones, complementarias, de Gómez de Somorrostro 1820, 215-225, y MASdeu 1800, 412 y ss. La intervención fue motivada por la llamada de atención que a Manuel Godoy realizaron (para que fuera puesto en conocimiento de Carlos IV), el cura de Duruelo, Santos Martín Sedeño, y el de Duratón, Cristóbal Rubio, en carta enviada al entonces duque de Alcudia el 18 de marzo de 1795, tras realizarse importantes descubrimientos en Los Mercados, en septiembre de 1791 y enero de 1795, cuando se había acudido al lugar con el objeto de recuperar material constructivo para restaurar la casa parroquial de Duratón. Ver: MARTínez CABALlERo 2010, 190 y ss.; ID. 2014. Sobre el paradero de los mosaicos (hoy en parte en el Museo Arqueológico Nacional), véase LuCAs Pellicer 1999.

4 Entre otros: CEÁN BERMúdez 1832, 187 (también señala la opción de identificar Confloenta con Segontia Lanca, siguiendo propuestas precedentes, en ediciones del siglo XVI de Ptolomeo y trabajos geográficos, de M. Villanovano, A. Hortelio, P. Ferrario y P. Bercio); Sentenach 1914, 9 (también cita Sepúlveda como alternativa a Duratón). 
también se colocaría Confloenta en la confluencia del Arlanzón con el Pisuerga. ${ }^{5}$ Por su parte, M. Cortés y López identificaba Confloenta con la Complega de Apiano (Iber. 42) y la situaba en Ágreda (Soria), y Govantes la ubicaba en Canales de la Sierra (La Rioja). ${ }^{6}$ Para Hübner la ciudad habría de enclavarse en una confluencia de ríos. Para A. Schulten y B. Taracena uno de estos sería el Duero. ${ }^{7}$ A. Molinero, como Comisario de Excavaciones de la Provincia de Segovia, excavaría desde 1941 en la necrópolis visigoda de Duratón y realizaría sondeos en la ciudad romana en 1949, junto a M. L. Déroche, de la Universidad de Bordeaux. ${ }^{8}$ Molinero identificaba Duratón con la Colenda de Apiano (Iber. 99) y, desde el hallazgo de materiales celtibéricos, formulaba la ocupación del lugar desde época prerromana. ${ }^{9}$

En el último cuarto del s. XX la evaluación del yacimiento de Los Mercados se restringe generalmente a considerar que el lugar solo debió acoger un asentamiento menor romano, ${ }^{10} \mathrm{y}$ recordar que Confloenta se localizaba en un emplazamiento concreto desconocido. ${ }^{11}$ No obstante, la importancia patrimonial del lugar se ve respondida a escala normativa con la declaración en 1994 del yacimiento como Bien de Interés Cultural, con categoría de Zona Arqueológica. ${ }^{12}$ Pero las nuevas prospecciones realizadas en Los Mercados en 1996 para el Inventario Arqueológico de Castilla y León no reconocieron la presencia de una ciudad romana, aunque, de forma paradójica, se considera que el sitio podría corresponderse con un municipio. ${ }^{13}$

\section{Identificación del municipium de Duratón desde las fuentes epigráficas}

Ya desde 1977 G. Alföldy, desde el análisis del cipo del Museo de Segovia, reflexionaba sobre el hecho de que Duratón hubiera constituido el centro político y administrativo de un municipio de derecho latino. ${ }^{14}$ Se trata de una inscripción en piedra cilíndrica, recuperada en Los Mercados y depositada en el Museo de Segovia en 1942, datada en 191 d.C., que recoge la mención de los seviri augustales, colegio sacerdotal presente en ciudades dotadas de estatuto jurídico privilegiado: L(ucius) Ter(entius)

5 MÜller 1883, 173. Recogido por Tovar 1989, 354. Para propuestas anteriores, ver supra n. 2.

6 Cortés y López 1836, 377-378; Govantes 1846, 47.

7 Hübner RE III, 873; Schulten 1914, 131; Taracena Aguirre 1954, 247, n. 13.

8 Molinero Pérez 1948; ID. 1949; ID. 1950; ID. 1971 e ID. 1979; Molinero Pérez - Linage Conde 1986.

9 Molinero Pérez 1950, 644; ID. 1952, 342 y ss.; ID. 1971, 136, lám XI. Barrio Martín 1999, 81-83, mantiene el argumento de situar un caserío prerromano de pequeñas dimensiones en Los Mercados.

10 García Merino 1975, 290; Zamora Canellada 1987, 45-48; Pernia Rodríguez - Veas Ruiz $1993,150$. Linage Conde 1972, 25, recoge la identificación de Confloenta con Duratón, pero considera que el núcleo político y administrativo de la zona se situaba en Sepúlveda.

11 Espinosa Ruiz 1984, 314; Salinas de Frías 1991, 214; Ramírez Sánchez 1997-1998, 73; Burillo Mozota 2007, 231 y 274.

12 Decreto 264/1994 de 24 de noviembre, publicado en el BOCYL, 3 de enero de 1995, 171-172. Atendiendo a la documentación de la prospección de García-Cruces et alii, ITAR, entre 1992 y 1993.

13 Junta de Castilla y Léon, Ficha no 40-195-0004-03, "Los Mercados". Prospección de Arquetipo S.L.

14 AlföLdy 1977. 
Sextio / L(uci) Ter(enti) Titia/ni lib(ertus) ob hon(orem) / sevir(atus) quem / gessit [[Aproni/ano]] et Maurico co(nsulibus). ${ }^{15}$

J. Santos Yanguas sumó a continuación al análisis la inscripción de Puente Talcano, junto a Sepúlveda, para incidir en la propuesta de colocar en Los Mercados la sede de un municipium de derecho latino. ${ }^{16}$ Conocida desde el s. XVIII, esta inscripción rupestre, colocada en este paraje junto al río Duratón, ${ }^{17}$ y datada el 24 de abril de 128 d.C., conmemora unos votos dedicados a Bonus Eventus por un ordo: Pro sal(ute) ordinis [....] / P(ublius) Val(erius) Natalis Maternia[ni] fil(ius) [c]um / suis [ar]am Eventu[i] Bono posuit et / dedicavit VIII k(alendas) Maias, A[sp]renate / Torquato II (= iterum) ma(iore) co(n)s(ule). Convivantes, / ite feliciter. ${ }^{18}$ El ordo debía ser el ordo decurionum de un municipium, el reconocido por el epígrafe anterior, pues Puente Talcano se localizaría en el territorium de Duratón.

Considerando estos documentos, R. Knapp planteaba la identificación de Los Mercados con la Nova Augusta arévaca de Plinio y Ptolomeo ${ }^{19}$ (si bien, de forma paralela se formulaba la identificación de esta última ciudad con el yacimiento de Lara de los Infantes, Burgos). ${ }^{20}$ Para ello Knapp se apoyaba en el texto de una inscripción de Sepúlveda (ERSg 163), en la que aparecía la secuencia [- - -]VST[- - ], que contendría el nombre de la ciudad.

En 1995 J. del Hoyo sumaba al argumento un fragmento de un texto en bronce recuperado en Los Mercados, perteneciente a una posible lex municipalis flavia, del municipio que habría de reconocerse en Duratón, aunque el autor no propone su nombre antiguo. Pero desecha el de Nova Augusta, desde la propuesta de identificación de esta última con Lara de los Infantes. ${ }^{21}$ La valoración de que en Duratón se debe reconocer un municipio romano comenzaría a recogerse desde la publicación de estos trabajos, ${ }^{22}$ aunque en los estudios arqueológicos no se asentara de forma paralela la consideración del lugar como ciudad romana. ${ }^{23}$ 22.

15 Santos Yanguas - Hoces de la Guardia Bermejo - Hoyo Calleja 2005 (en adelante ERSg), 90-92, nº

16 SAntos Yanguas 1985; Alföldy 1994.

17 Posiblemente se trata de un santuario rural dedicado a Bonus Eventus (Mangas Manjarrés - Martínez Caballero 2010, 345-347; Martínez Caballero 2014a.

18 ERSg 159.

19 Plin. NH 3.27; Ptol. 2.3.55; KNAPP 1992, 263.

20 Gimeno - MaYer 1993.

21 Hoyo Calleja 1995, 140-144 $(\rightarrow$ AE 1995, 862; HEp 6, 1996, 855 = Hoyo Calleja 1995a, 407-412, y lám. I.); ERSg 24.

22 Stylow 1990; Mangas Manjarrés 1996; García Fernández 2001; Andreu Pintado 2004; Hernández Guerra 2008.

23 Reseñamos el esfuerzo de Conte Bragado - Fernández Bernaldo de Quirós 1993, por volver a considerar la importancia del lugar. 


\section{Identificación de Confluenta con el municipium de Duratón: consolidación del argumento arqueológico y revisión del argumento literario}

Desde 2000 consideramos la presencia de una ciudad romana en Los Mercados con categoría de municipium. ${ }^{24}$ Entre 2001 y 2002 planteamos y llevamos a cabo un reconocimiento arqueológico del yacimiento, mediante sondeos de comprobación estratigráfica y microprospección, que tenían como objetivo la evaluación de las características y el estado de conservación de las estructuras arqueológicas del yacimiento, de cara a promover nuevas actuaciones de protección, puesta en valor e investigación, y comprobar el carácter urbano del enclave. Los trabajos confirmaron tal hipótesis. Detectamos su ocupación más antigua a inicios del s. I a.C. (el caserío prelatino está por comprobar), evidenciando que la ciudad se mantuvo como sede del poder administrativo local en toda la etapa imperial, como municipium desde época flavia (según la epigrafía), para pervivir como núcleo territorial principal hasta el s. V d.C. El lugar parece quedar abandonado entre la segunda mitad del s. VII e inicios del s. VIII d.C. ${ }^{25}$

Las prospecciones permiten considerar una superficie de la ciudad romana cercana a las 50 ha, aunque no toda fue ocupada en un mismo momento, existiendo además una variabilidad de ocupación zonal en función de la evolución cronológica. ${ }^{26}$ Por fotografía aérea se detecta un núcleo ordenado en función de ejes de planificación regular, que indican una ordenación original de nuevo cuño, propio de fundaciones ex novo, aunque sufre sucesivas ampliaciones, con posteriores variaciones de ejes de planificación. Los sondeos detectan estructuras de entidad monumental y compleja arquitectura, en especial el campus-forum pecuarium (con recinto emergente), la rampa de acceso a la ciudad y un espacio de tránsito de un edifico público (el excavado por Molinero y Deroché), quizás parte del visto en 1795, posiblemente unas termas centrales. Estas evidencias (a las que se suman una diversidad de materiales recuperados en el lugar: arquitectónicos, escultóricos, epigráficos -funerarios, votivos, jurídicos-, numismáticos, etc.), reconocen un espacio urbano dotado de soluciones urbanísticas, arquitectónicas y ornamentales habituales en las ciudades romanas de entidad y que alcanzaron un estatuto privilegiado en el valle del Duero.

El análisis arqueológico y de las fuentes literarias también nos ha permitido evaluar el origen de la ciudad romana. ${ }^{27} \mathrm{El}$ alto valle del Duratón fue conquistado por $T$. Didius (cos. 98 a.C.), entre 98 y 95 a.C. Ya que en 95 a.C. se enviaría una comisión senatorial (App., Iber. 100), para realizar las labores de reorganización de los territorios conquistados por Didio, que debieron incluir el valle alto del Duratón, pensamos que Didio y esta comisión debieron ser los responsables, por decisión tomada en el

24 Martínez Caballero 2000.

25 Sobre el urbanismo: Martínez Caballero - Mangas Manjarrés - Prieto Vázquez 2001; Martínez Caballero - Mangas Manjarrés 2002; Martínez Caballero - Prieto Vázquez - Orejas Saco del Valle 2004; Martínez Caballero 2008; ID. 2010; ID. 2011; ID. 2014a; ID. e.p.; ID. e.p. (a).

26 Orejas Saco del Valle - Martínez Caballero 2002.

27 Martínez Caballero 2008; ID. 2010; ID. 2010a; Martínez Caballero - Santiago Pardo 2010; Martínez Caballero 2011; ID. 2011a; Santos Yanguas - Martínez Caballero 2014; Martínez Caballero López Ambite - Gallego Revilla e.p. 
marco de la política de urbanización en el alto Duero, ${ }^{28}$ de la fundación de la nueva ciudad de Duratón, asentada en un llano, en la confluencia de los ríos Duratón y Serrano, ya que las prospecciones señalan que la primera ocupación urbana de Los Mercados se produce a inicios del s. I a.C. Esta fundación tenía como objetivo, al tiempo que inicialmente asegurar el control y seguridad militar del área, facilitar la gestión del territorio, un mejor acceso a las áreas de explotación y las comunicaciones, y ofrecer un emplazamiento apropiado para desarrollar las soluciones urbanísticas ajustadas al papel de la sede de una civitas. Se procedió a la centralización de las estructuras de poder y gestión en el valle del Duratón, con la reducción del número de unidades urbanas, derivando en una jerarquización más amplia del territorio y simplificación de la estructura territorial, desde la selección de la nueva posición en llano de Los Mercados como caput urbis de la civitas dediticia. La nueva fundación decidió el trasvase del principal componente demográfico del oppidum de Sepúlveda (transductio), que es anulado como centro de poder indígena, y se produjo al mismo tiempo la incorporación en el seno de su estructura cívica de nuevos aportes territoriales y demográficos, desde el desmantelamiento (puntual o progresivo) de los cercanos oppida segovianos de Los Quemados (Carabias), Morros de San Juan (Sebúlcor) y Los Sampedros (San Miguel de Bernuy) (¿mantienen población residual?). Se procedió al reemplazo de estructuras autónomas y la reordenación del territorio, desde la redistribución de propiedades, que consolidó una élite beneficiada de la misma y coadyuvó a la dinamización socioeconómica de la civitas. Esta actuación conllevó un gran impacto ideológico, pues era demostración de presencia y reelaboración de los mecanismos de comprensión del territorio, en detrimento de los marcos simbólicos de referencia indígenas. ${ }^{29}$

Tras una primera evaluación prudente, formulamos la propuesta de identificación de Los Mercados de Duratón con la Confloenta de Ptolomeo, que hemos mantenido en trabajos sucesivos, a partir de las evidencias enunciadas desde el análisis epigráfico y las características arqueológicas del lugar. ${ }^{30}$ Los datos arqueológicos que hemos extraído han ofrecido un parámetro sólido para apoyar esta propuesta, rescatando con ello la vieja hipótesis. ${ }^{31}$

La identificación del nombre de Duratón pasaba por atender principalmente el texto de Ptolomeo y otros que pudieran haberla mencionado en relación con ciudades arévacas citadas desde el s. I a.C. ${ }^{32}$ Belgeda es una ciudad de ubicación muy impre-

\footnotetext{
28 Martínez Caballero 2011a; Santos Yanguas - Martínez Caballero 2014.

29 Martínez Caballero 2008; ID. 2010; Santos Yanguas - Martínez Caballero 2014; Martínez Caballero - López Ambite - Gallego Revilla e.p.

30 Martínez Caballero - Prieto Vázquez - Orejas Saco del Valle 2004; Martínez Caballero 2008; ID. 2010; Mangas Manjarrés - Martínez Caballero 2010; Martínez Caballero - Santiago Pardo 2010; Martínez Caballero 2011; ID. 2011a; ID. 2013, Santos Yanguas - Martínez Caballero 2014; Martínez Caballero 2014a y e.p.; Martínez Caballero - López Ambite - Gallego Revilla e.p.

31 Santos Yanguas - Hoces de la Guardia Bermejo - Hoyo Calleja 2005, 19 y 78-82. La recopilación de NúÑEZ HERNÁNDEZ - CURCHIN 2007, 487-492, formula un reconocimiento del lugar, como centro urbano, desde nuestros textos, y mantiene el interrogante sobre la identificación de Duratón con Confluenta.

32 Como se deduce de la arqueología, el alto valle del Duratón formó parte del territorio arévaco. Ver: Barrio Martín 1999; Martínez Caballero 2000; Blanco García 2006; Martínez Caballero 2008; Martínez Caballero 2010a; ID. 2011a; López Ambite 2012; Martínez Caballero - López Ambite - Gallego Revilla
} 
cisa, citada en relación con las campañas de C. Valerius Flaccus (cos. 93 a.C.) en la Meseta Norte, en 93-92 a.C. (App., Iber. 100, lo que la alejaría del área de Duratón, conquistada con precedencia), o de la actuación de este magistrado en la Citerior celtibérica, en general. También es citada (Belgida) en 76 a.C., en las guerras sertorianas (Oros. 5.23.11). No está claro si se situaba en el valle del Duero, o en otro ámbito celtibérico del Tajo o el Ebro. Mutudureus y Meo[- - -] son ciudades conocidas en la guerra sertoriana, en 75-74 a.C. (Sal., Hist. 2.93). Su ubicación en el alto Duero es debatida, al igual que su etnicidad. Se descarta que alcanzaran el estatuto municipal en época imperial. Lo mismo sucede con Borvodurum, citada en una tésera del s. I a.C. de Vxama (Osma, Soria). Los editores consideran que puede o no localizarse en posición cercana a Vxama. Se desconoce si era una entidad urbana. ${ }^{33}$ Colenda es una ciudad conquistada después de Termes por el procónsul T. Didio, tras nueve meses de asedio (App., Iber. 99), en un momento indeterminado entre 97 y 95 a.C. Generalmente se considera ciudad celtíbera arévaca, proponiéndose su localización en los oppida arévacos de Sepúlveda, Ayllón o Los Quemados, o en la vaccea Cuéllar, en Segovia. ${ }^{34}$ Para nosotros podría ser la vettona Ulaca (Solosancho, Ávila) o la propia Sepúlveda arévaca, ${ }^{35}$ luego trasladada al emplazamiento de Los Mercados con la conquista romana, debiendo buscar el nombre de Duratón en otro topónimo. Se debe descartar su identificación con un municipium en Sepúlveda. ${ }^{36}$

Resta por atender al texto de Ptolomeo 2.6.55, donde quedaban por identificar Tucris y Confluenta de las diez póleis arévacas que el listado recoge..$^{37}$ En este punto hay que considerar los yacimientos arqueológicos que se corresponden con ciudades de época romana en el espacio arévaco y cuyo nombre antiguo se desconoce, para

e.p. Descartamos Axinio y Ocilis (App., Iber. 57), Malia (App., Iber. 76), Lagni (Diod. Sic. 33.17.1) y Lutia (App., Iber. 94) -síntesis en Burillo Mozota 2007-, por situarse en el alto Duero central y oriental.

33 Sobre Belgeda, síntesis en Burillo Mozota 2007. Lectura errónea de Mutudureus (como Mutudurum): Schulten 1933, Mutudurum; FHA IV, 220; Kroll 1933, Mutuduri; Tovar 1989; Pernia Rodríguez - Veas Ruiz 1993. Nueva lectura: Jordán Cólera 2001, 475-476. Sobre la tésera de Vxama: García Merino - Untermann 1999, sugieren que pudiera tratarse del oppidum de Gormaz, Soria. Para Jordán Cólera 2005, Borvodurum podría leerse Tarmodurum.

34 Sepúlveda: Sentenach 1914, 11; Molinero Pérez 1952, 342-343. Ayllón o Carabias: López Ambite 2012. Cuéllar: GómeZ de Somorrostro 1820; Wattenberg 1959; Barrio Martín 1999, 42-44.

35 En extenso, Martínez Caballero 2011a.

36 Como propone Hernández Guerra 2008, 437. Sepúlveda no constituyó ciudad ni cabeza de civitas en época imperial. Parece ser un lugar ya desocupado en época imperial (así lo confirman las excavaciones realizadas en Sepúlveda en los últimos años; Martínez CABALlero 2014); la presencia de un vicus es incluso dudable. La inscripción de Puente Talcano no apoya el reconocimiento de un municipium en Sepúlveda, sino en Duratón. Es evidente la falta de atención al modelo de poblamiento romano del área. Es inadmisible considerar que Duratón y Sepúlveda fueron ciudades romanas y municipia coexistentes. Se suma el uso de una metodología arbitraria en el análisis, como muestra un trabajo posterior (ver infra, n. 41), en el que el autor presta atención a la lectura de KNAPP 1992, 288, n 313, del epígrafe de San Frutos del Duratón, para apoyar el argumento de la municipalidad de Duratón, lo que es incongruente con desechar al mismo tiempo el epígrafe de Puente Talcano para el mismo fin, pues Puente Talcano se ubica en posición intermedia entre Los Mercados y San Frutos del Duratón. Además, se obvia la más reciente transcripción de ERSg 60, que desecha que en ese epígrafe de San Frutos se mencione la tribu Quirina.

37 Las posiciones de Clunia, Vxama, Termes, Numantia, Segovia, Nova Augusta y Segontia Lanca son bien conocidas. En Santos Yanguas - Martínez Caballero 2014, 464, proponemos identificar Veluca (la Voluce del It. Ant. 442.1), con Las Quintanas-Royo Albar (Quintana Redonda, Soria), entre Numantia y Vxama. 
tratar de reducir Tucris y Confloenta. Estos yacimientos son tres: Los Mercados de Duratón; Los Valladares-El Vadillo, ubicado cerca del río Duero, en Villalba, junto a Almazán (Soria), en el alto Duero central; y La Gotera, en Villaseca de Arciel (Soria), entre Numantia y Bilbilis, en el alto Duero oriental. El nombre de Confloenta parece latino, desde Confluenta/Confluentia, a relacionar con una condición topográfica de "confluencia". Los Mercados de Duratón surge en una confluencia de ríos, el Duratón y el Serrano, pero también de vías y caminos pecuarios: la vía del piedemonte del Sistema Central que, con sentido Este-Oeste, unía el alto Duero con las principales poblaciones situadas a los pies del piedemonte septentrional de la cordillera, desde Termes hasta Segovia y Avila, y Plasencia (coincidente con la actual N-110 entre Soria y Plasencia), también camino pecuario (reelaborado posteriormente en la Cañada Real Soriana Occidental); y la vía que unía ambas mesetas centrales hispanas por Somosierra, futura vía Complutum-Clunia, por donde transitaba otra vía pecuaria (luego la Cañada Real Segoviana). ${ }^{38}$ También se ha planteado que Confloenta derivaría, en cambio, de la latinización de un nombre céltico, del tipo Complutica o Compleutica, y desde el que se origina el topónimo de Complutum (cuya ceca prelatina es ikesankon konbouto); si bien, la etimología del término remitiría al mismo significado, ya que se ha planteado que Com-plou-to se explicaría desde "con-fluir", "acto de fluir juntos", con cognado griego (plšw) y germánico anglosajón (flówan "fluir"). ${ }^{39}$

La "confluencia" de ríos y comunicaciones son más evidentes en Los Mercados de Duratón que en las ciudades de Villalba y Villaseca de Arciel, donde no existe una posición destacada formulada desde la confluencia de ríos o de un nudo de comunicaciones tan evidente. Esa posición geográfica explica el potencial económico de la ciudad, volcado tanto a la explotación de la rica campiña del Duratón, como a la actividad ganadera y el tráfico de la sal, por gestionar un territorio rico en pastos y por ser lugar de tránsito de importantes vías pecuarias y de la vía de la sal que, procedente del centro productor de Segontia (Sigüenza, Guadalajara), abastecía de esta mercancía a la cuenca sedimentaria del Duero. Estas actividades empujaron las industrias artesanales y comerciales locales, potenciadas por la posición de la ciudad en ese cruce de caminos y también en el vado del Duratón, que orientó el trazado de la propia vía trazada por el piedemonte al norte del Sistema Central. Recordamos cómo importantes cabeceras de la Baetica (Hispalis, Italica, Corduba, Astigi, Ilipa...) se encuentran situadas junto a vados del Guadalquivir o de sus afluentes, al igual que en Lusitania, en el Guadiana (Emerita Augusta...), o en otras áreas de la Citerior, como en el Tajo (Complutum, Titulcia, Toletum, Caesaraugusta, Augustobriga...). ${ }^{40}$ Todos estos factores convirtieron a Duratón en uno de los focos de gravitación socioeconómica más destacados del sur del Duero, como escala de caminos y de actividad trashumante, y sede de un importante mercado regional (evocado todavía en la topografía, en el

38 El uso de este tipo de condiciones geográficas para elaborar un topónimo se observa en la Confluentes germana. Se trata de la posición donde confluyen el Rhin y el Mosela, donde Druso creó la posición fortificada de Castellum apud Confluentes en 9 a.C., germen de Confluentes, hoy Koblenz/Coblenza. La ciudad se colocó en la intersección de la vía del limes entre Mogontiacum (Mainz) y Colonia Ara Agrippinensium (Köln/ Colonia), y la del Mosela, procedente de Augusta Treverorum (Trier/Tréveris).

39 García Alonso 2003, 301.

$40 \mathrm{Al}$ respecto, Mangas Manjarrés e.p. 
nombre del actual yacimiento arqueológico), datos que explican la presencia de su amplio forum pecuarium, donde se acogerían mercados y ferias ganaderas y actividades comerciales y de celebración ligadas a las anteriores.

De otro lado, si bien las coordenadas señaladas por Ptolomeo contienen errores, ofrecen agrupaciones que permiten situar la posición relativa de algunas ciudades arévacas, por lo que su análisis podría aportar algún dato a tener en cuenta. Haciendo caso a este esquema, Confluenta habría de situarse entre Clunia, Termes y Vxama, en el área occidental arévaca, y en la misma longitud que Clunia, lo que se cumple en Duratón (habría error en la latitud). ${ }^{41}$

En suma, fruto de diferentes estudios realizados en los últimos años, la evaluación de la documentación literaria, epigráfica y arqueológica ofrece la conclusión de que la Confloenta de Ptolomeo, desde el topónimo latino Confluenta/Confluentia, se localiza en el yacimiento arqueológico de Los Mercados de Duratón. Solo falta su confirmación epigráfica.

Descartamos otra propuesta de identificar Duratón con la vaccea Porta Augusta de Ptolomeo, 2.6.49 (que se ha relacionado con un supuesto nombre de la Sepúlveda prerromana, Durato, hipótesis también a descartar), pues Duratón se ubica claramente en territorio arévaco. ${ }^{42}$ También, el cognomen leído como [D]uratonis de una inscripción de Monte Cildá, que se ha relacionado por algunos con Los Mercados, no es seguro. En cualquier caso, Duratonis documentaría un antropónimo desde un hidrónimo (Duratón, Durius), no desde un nombre antiguo de la ciudad, como el antropónimo Dureta, hapax documentado en León. ${ }^{43}$

\footnotetext{
41 No hemos incluido en bibliografía el texto de L. HeRnÁndez GuERRA, "Sobre la problemática ubicación de la ciudad de Confloenta", en C. Fernández y R. Bohigas, eds, In Durii Regione Romanitas. Estudios sobre la presencia romana en el valle del Duero en homenaje a Javier Cortés Álvarez de Miranda, Palencia/Santander 2012, 305-310. La sustancia de la argumentación y conclusión de este texto constituye una reproducción sin ninguna aportación nueva de nuestros trabajos, pero sin cita a los mismos. Ni siquiera se contiene que es nuestro equipo quien ha propuesto, desde la argumentación que tal autor utiliza, la conclusión que propone, a partir de la complementación del análisis epigráfico con el arqueológico. Nuestros datos, sin ser referenciados, son sintetizados y a veces directamente transcritos. La redacción ofrece dos apartados finales que, con textos desestructurados por mal uso de la materia de transcripción, reproduce los tomados directamente desde "La ciudad de Confluentia-Duratón y su territorio" (Martínez CABallero 2010). En suma, se construye, desde una argumentación ya expresada por nosotros, en anterior propuesta sobre la identificación de Confluenta con Duratón, una pretendida nueva propuesta de identificación de Confloenta con Duratón. Ni siquiera el adjetivo "problemático" que incluye en el título del artículo procede, cuando con precedencia ya hemos aportado los argumentos, soluciones y conclusiones a las cuestiones que el autor se pregunta.

42 Según Rodríguez Morales 2005. Porta Augusta se localiza en el área septentrional del Duero medio (síntesis en García Alonso 2003, 257-258). Se propone que el topónimo estaría basado en la posición de Duratón a los pies del paso de Somosierra, "puerta" de la Meseta Norte. Se formularía desde el topónimo céltico de Sepúlveda, que sería Durato, desde el duratin de una tésera de hospitalidad de Ávila [CT-6], de la colección Turiel, relacionable con el indoeuropeo dhur-, "puerta". BeLtrÁn LloRis - JORDÁN CólerA - Simón CornaGo 2009, 630, señalan que [CT-6] es muy sospechosa, tosquísima y con un rótulo de aspecto ibérico, por lo que no debe ser tenida en cuenta (los análisis metalográficos, iconográficos y epigráficos han revelado la falsedad de [CT-4, 7, 12, 13, 14, 15, 16, 17 y 18] de la misma colección).

43 Ramírez Sádaba - Campos Lastra 2010, 454-455. Puede corresponder también a Uratonis. Dureta: véase IRPle 194 (Diego SANTos 1986).
} 


\section{Conclusiones}

Las investigaciones que hemos llevado a cabo en el yacimiento de Los Mercados de Duratón han confirmado la presencia en el lugar de una ciudad romana, en la que se hacen presentes componentes urbanísticos y arquitectónicos ajustados a los detectados en los enclaves urbanos principales y con estatuto jurídico privilegiado del valle del Duero. Los datos epigráficos permiten identificar en Duratón, según venían señalando otros autores (Alföldy, Santos, Knapp, y del Hoyo), el caput civitatis de un municipium de derecho latino. El análisis de estos datos, en conjunto con la atención a Ptolomeo 2.6.55, nos ha permitido proponer con sólidos argumentos en los años 2000 la identificación de esta ciudad y municipium con la pólis celtíbero-arévaca de Confloenta. Se trata de una ciudad fundada a inicios del s. I a.C., actuación enmarcada en la política romana de urbanización y reestructuración territorial aplicada tras la conquista, fundamentada en la desarticulación del oppidum de Sepúlveda y su traslado (transductio), como la mayor parte de su componente poblacional, a la ciudad de Confloenta, caput urbis de la nueva civitas dediticia desde la que se estructuró la gestión y ordenación del territorio del alto Duratón en lo sucesivo, y ciudad que adquirirá el estatuto municipal en época flavia y mantendrá su carácter urbano hasta época tardo-antigua.

\section{BIBLIOGRAFÍA}

AlFöLDY, G.

(1977): "Eine Römische Inschrift aus Duraton in der Hispania Citerior”, ZPE 27, 222-228.

(1994): "Epigraphica Hispanica XV: Eine Felsinschrift bei Sepúlveda (prov. Segovia)", ZPE 100, 451-463.

Andreu Pintado, J. (2004): Edictum, Municipium y Lex: Hispania en época flavia (69-96 d.C.), (=BAR International Series 1293), Oxford.

Barrio Martín, J. (1999): La II Edad del Hierro en Segovia (España). Estudio arqueológico del territorio y la cultura material de los pueblos prerromanos (=BAR International Series 790), Oxford.

Beltrán Lloris, F. - Jordán Cólera, C. - Simón Cornago, I. (2009): “Revisión y balance de téseras celtibéricas", [en] F. Beltrán et alii (eds.), Acta Palaeohispanica X. Actas do X Colóquio sobre Línguas e Culturas Paleo-hispânicas (Lisboa, 26-28 de fevereiro de 2009), (=Palaeohispanica 9), Zaragoza, 625-668.

Blanco García, J. F.

(1998): “La Edad del Hierro en Sepúlveda (Segovia)”, Zephyrus LI, 137-174.

(2006): "El paisaje poblacional segoviano en época prerromana: ocupación del territorio y estrategias de urbanización", Oppidum 2, 35-84.

Burillo Mozota, F. (2007): Celtíberos. Etnias y estados, Barcelona.

Cé́n Bermúdez, J. (1832): Sumario de las antigüedades romanas que hay en España. En especial las pertenecientes a las Bellas Artes, Madrid. 
Conte Bragado, D. - Fernández Bernaldo de Quirós, I. (1993): Introducción a la arqueología en el cañón del Duratón, Segovia.

Córnide, J. (1799): "Noticia de las antigüedades de Cabezo de Griego, reconocidas de orden de la Real Academia de la Historia”, Memorias de la Real Academia de la Historia III, 71-244.

Cortés y López, M. (1836): Diccionario geográfico-histórico, Madrid, vol. III.

Diego SANTos, F. (1986): Inscripciones romanas de la provincia de León, León (=IRPLe).

Espinosa Ruiz, U. (1984): "Las ciudades de arévacos y pelendones en el Alto Imperio. Su integración jurídica", [en] C. de la Casa Martínez (coord.), Actas del I Symposium de arqueología Soriana, Soria, 307-324.

García Alonso, J. L. (2003): La Península Ibérica en la Geografía de Claudio Ptolomeo (=Anejos de Veleia, Serie minor 19), Vitoria-Gasteiz.

García Fernández, E. (2001): El municipio latino. Origen y desarrollo constitucional (=Gerión, Anejos V), Madrid.

García Merino, C. (1975): Población y poblamiento en Hispania romana. El Conventus Cluniensis, Valladolid.

García Merino, C. - Untermann, J. (1999): "Revisión de la lectura de la tessera uxamensis y valoración de las téseras en el contexto de la configuración del poblamiento celtibérico en el s. I a.C.”, Boletín del Seminario de Estudios de Arte y Arqueología LXV, 138-152.

Gimeno, H. - Mayer, M. (1993): "Una propuesta de identificación epigráfica. Lara de los Infantes / Nova Augusta", Chiron 23, 313-321.

Ginés de Sepúlveda, J. (1571): De Regno et regis officiis, Madrid.

Gómez de Somorrostro, A. (1820): El Acueducto y otras antigüedades de Segovia, Madrid.

Govantes, A. C. DE (1846): Diccionario Geográfico-Histórico de España, Madrid.

Grutero, J. (1603): Inscriptiones antiquae totius orbis Romani in corpus absolutissimum redactae, Heidelberg.

Hernández Guerra, L. (2008), “Algunas consideraciones sobre municipios Flavios en la Meseta Septentrional”, Gerión 26/1, 407-438.

Hoyo Calleja, J. Del

(1995): "Duratón, municipio romano. A propósito de un fragmento inédito de ley municipal", ZPE 108, 140-144.

(1995a): "Duratón, municipio romano. A propósito de un fragmento inédito de ley munici-

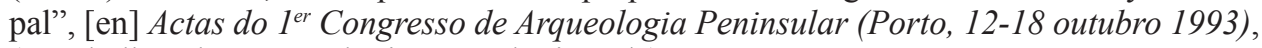
(=Trabalhos de Antropologia e Etnologia 35/3), Porto, 407-412.

JoRDÁn CÓleRA, C.

(2001): "Sobre la etimología de Botorrita y su confirmación en la onomástica prelatina", [en] F. Villar - M M $^{\mathrm{a}}$. Fernández Álvarez (eds.), Religión, Lengua y Cultura Prerromanas de Hispania. VII Coloquio sobre Lenguas y Culturas Prerromanas de la Península Ibérica (=Acta Salmanticensia, Estudios Filológicos 283), Salamanca, 471-480.

(2005): “¿Sistema dual en celtibérico?”, [en] F. Beltrán - C. Jordán - J. Velaza (eds.), Acta Paleohispanica IX. Actas del IX Coloquio Internacional sobre Lenguas y Culturas Paleohispánicas (Barcelona, 2004), (=Palaeohispanica 5), Zaragoza, 1013-1030.

KNAPP, R. C. (1992): Latin Inscriptions from Central Spain (=Classical Studies 34), Berkeley-Los Angeles-Oxford (=LICS). 
Kroll, W. (1933): "Mutuduri”, [en] Realencyclopädie der classischen Altertumswissenschaft Band XVI. Halbband 31, Molatzes-Myssi, Berlin, 979.

Linage Conde, A. (1972): Hacia una biografía de la villa de Sepúlveda, Segovia.

LoperrÁEz, J. (1788): Descripción histórica del Obispado de Osma con el catálogo de sus prelados, Madrid, tomo I.

López Ambite, F. (2012): El poblamiento en la periferia de la cuenca del Duero. El nordeste de la provincia de Segovia. España (XII cal. A.C.-V d.C.), (=BAR International Series 2323), Oxford.

Lucas Pellicer, Ma R. (1999): "Mosaicos del sitio de "Los Mercados” en Duratón (Segovia)", [en] J. Alvar (ed.), Homenaje a José Ma Blázquez, Madrid, vol. IV, 275-287.

MANGAS ManjarRés, J.

(1996): "Derecho latino y municipalización de la Meseta Superior", [en] E. Ortiz - J. Santos (eds.), Teoría y práctica del ordenamiento municipal en Hispania. Actas del Symposium de Vitoria-Gasteiz (22 a 24 de noviembre de 1993), (=Veleia, Anejos, Series Acta 3), Vitoria-Gasteiz, 223-238.

(e.p.): "Vados, poblados, vías, ladrones y dioses: Alto y Medio Tajo", [en] Homenaje a Filippo Coarelli.

Mangas Manjarrés, J. - Martínez Caballero, S. (2010): “La religión de la población del territorio segoviano en época romana", [en] Martínez Caballero - Santiago Pardo - Zamora Canellada (coords.), 2010, 335-358.

Martín Aymerich, Ma D. - Tardío Dovao, T. - Zamora Canellada, A. (1990): las murallas de Sepúlveda (Segovia). Un ensayo de aproximación con métodos arqueológicos a un ejemplo de pervivencia arquitectónica, Segovia.

Martínez Caballero, $\mathrm{S}$.

(2000): "Notas sobre la romanización del territorio segoviano", [en] VV. AA., Segovia romana, Segovia, 11-42.

(2008): "Los territorios de los municipios del Sur del Conventus Cluniensis (Hispania Citerior) en el Alto Imperio: Termes, Duratón y Segovia", [en] J. Mangas - M. Á. Novillo (eds.), El territorio de las ciudades romanas, Madrid, 187-247.

(2010): "La ciudad de Confluentia-Duratón y su territorio", [en] Martínez Caballero - Santiago Pardo - Zamora Canellada (coords.), 2010, 183-219.

(2010a): "El territorio segovianos entre la conquista romana y el fin de la República", [en] Martínez Caballero - Santiago Pardo - Zamora Canellada (coords.), 2010, 39-74.

(2011): "El territorio de Sepúlveda en época romana: la ciudad de Duratón-Confluentia", [en] M. González - G. Herrero - A. Linage (coords.), Historia de Sepúlveda, Segovia, 83-128.

(2011a): "La ciudad fundada por M. Marius, Termes y Colenda (App., Iber. 99-100). La guerra de 104-93 a.C. en territorios arévacos, vacceos y vettones", Studia Historica. Historia Antigua 29, 119-151.

(2013): "Forum, forum pecuarium y campus en las ciudades romanas de Confluentia-Duratón y Termes", [en] R. M ${ }^{a}$ Cid - E. García (eds.), Debita verba. Homenaje al Profesor Julio Mangas, Oviedo, vol. I, 69-92.

(2014): Confloenta. La ciudad romana de Duratón, (Sepúlveda, Segovia), Segovia.

(2014a): "Bonus Eventus en Puente Talcano (Sepúlveda, Segovia). Un santuario rural en el territorio del municipium de Duratón (Hispania Citerior)", [en] J. Mangas Manjarrés - M. Á. Novillo López (eds.), Santuarios suburbanos y del territorio en las ciudades romanas, Madrid, 339-382. 
(e.p.): "Evolución urbana y difusión de los modelos arquitectónicos romanos en la ciudad de Confluentia (Duratón, Segovia, Hispania Citerior)", [en] XVIII Congreso Internacional de Arqueología Clásica. Centro y periferia en el mundo clásico. Mérida, 13-17 de Mayo, 2013. (e.p., a): "El campus-forum pecuarium de Confloenta", [en] Los espacios de reunión de las asociaciones romanas. Diálogos desde la arqueología y la historia, en homenaje a Bertrand Goffaux, Sevilla.

Martínez Caballero, S. - López Ambite, F. - Gallego Revilla, J. I. (e.p.): “Tiermes y el área arévaca occidental. Ss. IV a.C.-I d.C. La Protohistoria como modelo de frontera", [en] F. Burillo (ed.), VII Simposio sobre celtíberos nuevos hallazgos, nuevas interpretaciones (Daroca, 20-22 de marzo de 2012).

Martínez Caballero, S. - Mangas Manjarrés, J. (2002): Informe. Excavaciones arqueológicas en Duratón. Campaña 2002, Junta de Castilla y León.

Martínez Caballero, S. - Mangas Manjarrés, J. - Prieto Vázquez G. (2001): Informe. Excavaciones arqueológicas en Duratón. Campaña 2001, Junta de Castilla y León.

Martínez Caballero, S. - Prieto VÁzquez, G. (2002): "Duratón romano: El yacimiento arqueológico de Los Mercados de Duratón”, Historia Digital, publicación on-line.

Martínez Caballero, S. - Prieto Vázquez, G. - Orejas Saco del Valle, A. (2004): “Duratón, ciudad romana", RevArq 272, 46-53.

Martínez Caballero, S. - Santiago Pardo, J. (2010): "La ocupación del territorio segoviano en época imperial romana (ss. I-V d.C.)", [en] Martínez Caballero - Santiago Pardo - Zamora Canellada (coords.), 2010, 75-118.

Martínez Caballero, S. - Santiago Pardo, J. - Zamora Canellada, A. (Coords.), (2010): Segovia romana II. Gentes y territorios, Segovia.

Masdeu, J. F. (1800): Historia Crítica de España y de la Cultura Española, Madrid.

Mayans, G. (1779): Tractatus de hispana progenie vocis ur, Madrid.

Molinero Pérez, A.

(1948): La necrópolis visigoda de Duratón (=Acta Arqueológica Hispánica 4), Madrid.

(1949): Excavaciones arqueológicas antiguas y modernas en Duratón (Segovia), Segovia. (1950): "Diez Años de Arqueología Segoviana", ES 5-6, 641-653.

(1952): "Una necrópolis del hierro céltico en Cuéllar (Segovia)", [en] II Congreso Nacional de Arqueología. (Madrid, 1951), Zaragoza, 337-354.

(1971): Aportación de las excavaciones y hallazgos casuales (1941-1959) al Museo de Segovia (=Excavaciones Arqueológicas en España 71), Madrid.

(1979): Un hecho digno de ser recordado en su primer cincuentenario: el descubrimiento de la necrópolis visigoda de Duratón (1929-1979), Segovia.

Molinero Pérez, A. - Linage Conde, A. (1986): "Unas excavaciones romanas inéditas de 1949 en el pueblo de Duratón (Segovia), Sepúlveda”, [en] Homenaje a Pedro Sáinz Rodríguez. 1.- Repertorios, textos y comentarios, Madrid, 235-247.

Morales, A. DE (1575): Las Antigüedades de las ciudades de España: que van nombradas en esta cronica, con la averiguación de sus sitios y nombres antiguos que escrevia, Alcalá de Henares.

Müller, K. (1883): Cl. Ptolomei Geographia, Paris.

NúÑEz Hernández, S. - Curchin, L. A. (2007): “Corpus des villes. Corpus de ciudades romanas en el valle del Duero", [en] M. Navarro - J. J. Palao (eds.), Villes et térritoires dans le bassin du Douro à l'époque romaine (=Ausonius Éditions, Études 17), Bordeaux, 429-612. 
Orejas Saco del Valle, A. - Martínez Caballero, S. (2002): Informe de las prospecciones arqueológicas en Los Mercados de Duratón (Sepúlveda, Segovia), Junta de Castilla y León.

Pernia Rodríguez, A. - Veas Ruiz, N. (1993): "Mercados, Los", [en] Unión académica internacional, Comité español, Tabula Imperii Romani. Hoja K-30: Madrid. Caesaraugvsta - Clvnia, Madrid, 150.

Ramírez SÁdABA, J. L. - CAmpos Lastra, R. (2010): “Cautelas sobre los nombres personales documentados una sola vez: el ara dedicada a Cabuniegino", Palaeohispanica 10, 447-449.

Ramírez SÁnchez, M. (1997-1998): “Los núcleos de población de las comunidades indígenas del Alto Duero a través de las fuentes literarias y arqueológicas", Vegueta 3, 65-87.

Rodríguez Morales, F. J. (2005): "La divisoria de los términos de las ciudades del centro de la Península en época romana y su posterior perduración”, [en] G. Bravo Castañeda - R. González Salinero (coords.), La aportación romana a la formación de Europa: naciones, lenguas y culturas. Actas del II Coloquio de la AIER (=Signifer, Monografías y Estudios de Antigüedad Griega y Romana 16), Madrid, 105-140.

Rodríguez VAlCÁRcel, F. (1647): Epitome Iuris Canonici, Alcalá de Henares.

Salinas De FríAs, M. (1991): “Arévacos”, [en] J. M. Solana (ed.), Las entidades étnicas de la Meseta Norte de Hispania en Época Prerromana (=Hispania Antiqua, Anejos 15), Valladolid.

Santos Yanguas, J. (1985): "La inscripción de Puente Talcano, Sepúlveda, Segovia (CIL II 5095=3089). Nueva lectura e interpretación”, [en] Symbolae L. Mitxelena septuagenario oblatae (=Anejos de Veleia 1), Vitoria-Gasteiz, 537-545.

Santos Yanguas, J. - Hoces de la Guardia Bermejo, Á. L. - Hoyo Calleja, J. del (2005): Epigrafía romana de Segovia y su provincia, Segovia (=ERSg).

Santos Yanguas, J. - Martínez Caballero, S. (2014): “Modelos de urbanización en época republicana en la Celtiberia de arévacos y pelendones", [en] Hoc qvoqve laboris paremium. Studi in onore di Gino Bandelli, Trieste, 457-476.

Schulten, A.

(1914): Numantia. Die Ergebnisse der Ausgrabungen 1905-1912 I. Die Keltiberer und ihre Kriege mit Rom, München.

(1933): "Mutudurum", [en] Realencyclopädie der classischen Altertumswissenschaft Band XVI. Halbband 31, Molatzes-Myssi, Berlin, 979.

Sentenach, N. (1914): “Los Arévacos”, RABM XXX, 1-22 y 181-200.

Stylow, A. U. (1990): "Neue Inschriften aus Carpetanien", Chiron 20, 317-323.

Taracena Aguirre, B. (1954): “Los pueblos celtibéricos”, [en] R. Menéndez Pidal (dir.), Historia de España, Madrid, tomo I, vol. 3, 238-242.

Tovar, A. (1989): Iberische Landeskunde. III. Tarraconensis, Baden-Baden.

Traggia, J. DE (1752): Aparato de Historia Eclesiástica de Aragón, Madrid, tomo II.

Vaillant, J. F. (1688): Numismata aerea Imperatorum, Augustarum et Caesarum in coloniis, municipiis, et urbibus jure Latio donatis, ex omni modulo percussa, Paris.

Wattenberg, F. (1959): La región vaccea. Celtiberismo y romanización en la Cuenca Media del Duero (=Bibliotheca Praehistorica Hispana II), Madrid.

Zamora Canellada, A. (1987): "Segovia en la Antigüedad", [en] Historia de Segovia, Segovia, $45-48$. 